Acta Univ. Sapientiae, Mathematica, 5, 1 (2013) 83-92

DOI 10.2478/ausm-2014-0006

\title{
About a condition for starlikeness
}

\author{
Róbert Szász \\ Sapientia Hungarian University of \\ Transylvania \\ Department of Mathematics and \\ Informatics \\ Târgu Mureş, Romania \\ email: rszasz@ms.sapientia.ro
}

\author{
Pál Kupán \\ Sapientia Hungarian University of \\ Transylvania \\ Department of Mathematics and \\ Informatics \\ Târgu Mureş, Romania \\ email: kupanp@ms.sapientia.ro
}

Dedicated to the memory of Professor Antal Bege

\begin{abstract}
A result concerning the starlikeness of the image of the Alexander operator is improved in this paper. The techniques of differential subordinations are used.
\end{abstract}

\section{Introduction}

Let $\mathrm{U}\left(z_{0}, \mathrm{r}\right)=\left\{z \in \mathbb{C}|| z-z_{0} \mid<\mathrm{r}\right\}$ be the disk centred in $z_{0}$ and let $\mathrm{U}=\mathrm{U}(0,1)$ be the open unit disk in $\mathbb{C}$. Let $\mathcal{A}$ be the class of analytic functions $\mathrm{f}$, which are defined on the unit disc $U$ and have the form: $f(z)=z+a_{2} z^{2}+a_{3} z^{3}+\cdots$.

The subclass of $\mathcal{A}$ consisting of functions for which the domain $\mathrm{f}(\mathrm{U})$ is starlike with respect to 0 , is denoted by $\mathrm{S}^{*}$. An analytic characterization of $\mathrm{S}^{*}$ is given by

$$
\mathrm{S}^{*}=\left\{\mathrm{f} \in \mathcal{A}: \mathfrak{R} \frac{z \mathrm{f}^{\prime}(z)}{\mathrm{f}(z)}>0, z \in \mathrm{U}\right\} .
$$

Another subclass of $\mathcal{A}$ we deal with is the class of close-to-convex functions denoted by $\mathrm{C}$. A function $\mathrm{f} \in \mathcal{A}$ belongs to the class $\mathrm{C}$ if and only if there is a starlike function $\mathrm{g} \in \mathrm{S}^{*}$, so that $\mathfrak{R} \frac{z \mathrm{f}^{\prime}(z)}{\mathrm{g}(z)}>0, \quad z \in \mathrm{U}$. We note that $\mathrm{C}$ and

Key words and phrases: Alexander operator, starlike functions, close-to-convex functions 
$S^{*}$ contain univalent functions. The Alexander integral operator is defined by the equality

$$
A(f)(z)=\int_{0}^{z} \frac{f(t)}{t} d t
$$

The authors of [1] (p. 310-311) proved the following result:

Theorem 1 Let $\mathrm{A}$ be Alexander operator and let $\mathrm{g} \in \mathcal{A}$ satisfy

$$
\mathfrak{R} \frac{z g^{\prime}(z)}{g(z)} \geq\left|\mathfrak{I} \frac{z\left(z g^{\prime}(z)\right)^{\prime}}{g(z)}\right|, z \in \mathrm{U} \text {. }
$$

If $\mathrm{f} \in \mathcal{A}$ and

$$
\mathfrak{R} \frac{z f^{\prime}(z)}{g(z)}>0, z \in \mathrm{U}
$$

or

$$
\mathfrak{R} \frac{f^{\prime}(z)}{g^{\prime}(z)}>0, z \in \mathrm{U}
$$

then $\mathrm{F}=\mathrm{A}(\mathrm{f}) \in \mathrm{S}^{*}$.

In [1], [3], [5] improvements of the first part ((1), (2) $\left.\Rightarrow A(f) \in S^{*}\right)$ of this result is proved, simplifying condition (1). The aim of this paper is to give an improvement for the second part of Theorem 1. In order to do this, we need the definitions and lemmas exposed in the next section.

\section{Preliminaries}

Let $f$ and $g$ be analytic functions in $\mathrm{U}$. The function $f$ is said to be subordinate to $g$, written $f \prec g$, if there is a function $w$ analytic in $U$, with $w(0)=0$, $|w(z)|<1, z \in U$ and $f(z)=g(w(z)), z \in U$. Recall that if $g$ is univalent, then $f \prec g$ if and only if $f(0)=g(0)$ and $f(U) \subset g(U)$.

Lemma 1 [2] p. 24 (Miller-Mocanu)

Let $\mathrm{p}(\mathrm{z})=\mathrm{a}+\sum_{\mathrm{k}=\mathrm{n}}^{\infty} \mathrm{a}_{\mathrm{k}} z^{\mathrm{k}}$ be analytic in $\mathrm{U}$ with $\mathrm{p}(z) \not \equiv \mathrm{a}, \mathrm{n} \geq 1$ and let $\mathrm{q}: \mathrm{U} \rightarrow \mathbb{C}$ be an analytic and univalent function with $\mathrm{q}(0)=\mathrm{a}$. If $\mathrm{p}$ is not subordinate to $\mathrm{q}$, then there are two points $z_{0} \in \mathrm{U},\left|z_{0}\right|=r_{0}$ and $\zeta_{0} \in \partial \mathrm{U}$ and a real number $\mathrm{m} \in[\mathrm{n}, \infty)$, so that $\mathrm{q}$ is defined in $\zeta_{0}, \mathrm{p}\left(\mathrm{U}\left(0, \mathrm{r}_{0}\right)\right) \subset \mathrm{q}(\mathrm{U})$, and: 
(i) $\mathrm{p}\left(z_{0}\right)=\mathrm{q}\left(\zeta_{0}\right)$,

(ii) $z_{0} p^{\prime}\left(z_{0}\right)=m \zeta_{0} q^{\prime}\left(\zeta_{0}\right)$,

and

(iii) $\operatorname{Re}\left(1+\frac{z_{0} p^{\prime \prime}\left(z_{0}\right)}{p^{\prime}\left(z_{0}\right)}\right) \geq \operatorname{mRe}\left(1+\frac{\zeta_{0} q^{\prime \prime}\left(\zeta_{0}\right)_{0}}{q^{\prime}\left(\zeta_{0}\right)}\right)$.

We note that $z_{0} p^{\prime}\left(z_{0}\right)$ is the outward normal to the curve $\mathrm{p}\left(\partial \mathrm{u}\left(0, \mathrm{r}_{0}\right)\right)$ at the point $\mathrm{p}\left(z_{0}\right)$. ( $\partial \mathrm{U}\left(0, \mathrm{r}_{0}\right)$ denotes the border of the disc $\mathrm{U}\left(0, \mathrm{r}_{0}\right)$.)

Lemma 2 [2] p. 26 (Miller-Mocanu) Let $p(z)=a+\sum_{k=n}^{\infty} a_{k} z^{k}, p(z) \not \equiv a$ and $\mathrm{n} \geq 1$.

If $z_{0} \in \mathrm{U}$ and

$$
\operatorname{Re} p\left(z_{0}\right)=\min \left\{\operatorname{Re} p(z):|z| \leq\left|z_{0}\right|\right\}
$$

then and

(i) $z_{0} p^{\prime}\left(z_{0}\right) \leq-\frac{n}{2} \frac{\left|p\left(z_{0}\right)-a\right|^{2}}{\operatorname{Re}\left(a-p\left(z_{0}\right)\right)}$

(ii) $\operatorname{Re}\left[z_{0}^{2} p^{\prime \prime}\left(z_{0}\right)\right]+z_{0} p^{\prime}\left(z_{0}\right) \leq 0$.

Lemma 3 If $\mathrm{d}=\frac{2}{\pi} \arctan \left(\frac{1}{2.273}\right)$, and $\mathrm{k}_{\mathrm{d}}(z)=\int_{0}^{z} \frac{\left(\frac{1+\mathrm{t}}{1-\mathrm{t}}\right)^{\mathrm{d}}-1}{\mathrm{t}} \mathrm{dt}$, then

$$
\left|\mathfrak{I}\left(\mathrm{k}_{\mathrm{d}}(z)\right)\right| \leq \frac{\pi}{6}, \quad z \in \mathrm{U} .
$$

Proof. The maximum principle for harmonic functions implies that

$$
\sup _{z \in \mathrm{U}}\left|\mathfrak{I} k_{\mathrm{d}}(z)\right|=\sup _{\theta \in[-\pi, \pi]}\left|\mathfrak{I} k_{\mathrm{d}}\left(e^{i \theta}\right)\right| \text {. }
$$

On the other hand we have:

$$
v_{d}(\theta)=\Im k_{d}\left(e^{i \theta}\right)=\int_{0}^{1} \frac{1}{x}\left(\frac{1+x^{2}+2 x \cos \theta}{1+x^{2}-2 x \cos \theta}\right)^{d} \sin \left(d \arctan \left(\frac{2 x \sin \theta}{1-x^{2}}\right)\right) d x .
$$

This implies that $v_{n}$ is an even function, consequently

$$
\sup _{\theta \in[-\pi, \pi]}\left|\mathfrak{I} k_{\mathrm{d}}\left(e^{i \theta}\right)\right|=\sup _{\theta \in[0, \pi]}\left|\mathfrak{I} k_{\mathrm{d}}\left(e^{i \theta}\right)\right| .
$$

We will prove the following equality:

$$
\begin{array}{r}
k_{d}\left(e^{i \theta}\right)=\int_{0}^{1} \frac{\left(\frac{1+x e^{i \theta}}{1-x e^{i \theta}}\right)^{d}-1}{x} d x=\int_{0}^{\infty}\left[\left(\frac{e^{t}-1}{e^{t}+1}\right)^{d}-1\right] d t+i(\pi-\theta)+ \\
\left(\sin \left(\frac{\pi}{2} d\right)-i \cos \left(\frac{\pi}{2} d\right)\right) \int_{0}^{\pi-\theta} \tan ^{d} \frac{x}{2} d x, \theta \in[0, \pi] .
\end{array}
$$


We begin with the observation that the change of variable $x=e^{-t}$ leads to

$$
k_{d}\left(e^{i \theta}\right)=\int_{0}^{\infty}\left[\left(\frac{e^{t}+e^{i \theta}}{e^{t}-e^{i \theta}}\right)^{d}-1\right] d t .
$$

Let $\theta \in[0, \pi]$ and consider the function

$$
f(z)=\left(\frac{e^{z}+e^{i \theta}}{e^{z}-e^{i \theta}}\right)^{d}-1 .
$$

We integrate it on $\Gamma=\gamma_{1} \cup \gamma_{2} \cup \gamma_{3} \cup \gamma_{4}$, where $\gamma_{1}(t)=t, t \in[0, R], \gamma_{2}(t)=$ $\mathrm{R}-\mathrm{it}, \mathrm{t} \in[0, \pi-\theta], \gamma_{3}(\mathrm{t})=\mathrm{R}-\mathrm{t}+\mathrm{i}(\theta-\pi), \mathrm{t} \in[0, \mathrm{R}]$ and $\gamma_{4}(\mathrm{t})=$ $i(\theta-\pi+t), t \in[0, \pi-\theta]$. The obtained equality $\int_{\Gamma} f(z) d z=0$ leads to

$$
\begin{array}{r}
k_{d}\left(e^{i \theta}\right)=\lim _{R \rightarrow \infty} \int_{\gamma_{1}} f(z) d z=-\lim _{R \rightarrow \infty}\left[\int_{\gamma_{2}} f(z) d z+\int_{\gamma_{3}} f(z) d z+\right. \\
\left.\int_{\gamma_{4}} f(z) d z\right]=\int_{0}^{\infty}\left[\left(\frac{e^{x}-1}{e^{x}+1}\right)^{d}-1\right] d x+ \\
i(\pi-\theta)+\left(\sin \left(\frac{\pi}{2} d\right)-i \cos \left(\frac{\pi}{2} d\right)\right) \int_{0}^{\pi-\theta} \tan ^{d} \frac{x}{2} d x .
\end{array}
$$

Thus, it follows

$$
\nu_{\mathrm{d}}(\theta)=\Im k_{\mathrm{d}}\left(e^{i \theta}\right)=\pi-\theta-\cos \left(\frac{\pi}{2} \mathrm{~d}\right) \int_{0}^{\pi-\theta} \tan ^{\mathrm{d}} \frac{\chi}{2} \mathrm{~d} x .
$$

The function $v_{\mathrm{d}}:[0, \pi] \rightarrow \mathbb{R}$ has a maximum at the point $\theta_{\mathrm{d}}=2 \arctan$ $\left(\cos ^{\frac{1}{d}}\left(\frac{\pi}{2} d\right)\right)$. A suitable numerical approach shows that

$$
\left|\Im\left(k_{d}(z)\right)\right| \leq v_{d}\left(\theta_{d}\right)=0.49 \cdots<\frac{\pi}{6}
$$

Lemma 4 If $\mathrm{q}_{\mathrm{d}}(z)=\exp \left(\int_{0}^{z} \frac{\left(\frac{1+\mathrm{t}}{1-\mathrm{t}}\right)^{\mathrm{d}}-1}{\mathrm{t}} \mathrm{dt}\right)=\exp \left(\mathrm{k}_{\mathrm{d}}(z)\right), \mathrm{p} \in \mathcal{A}$, and

$$
\frac{z p^{\prime}(z)}{p(z)} \prec h(z)=\frac{z q_{d}^{\prime}(z)}{q_{d}(z)}, z \in \mathrm{U},
$$

then $\mathrm{p} \prec \mathrm{q}_{\mathrm{d}}$. 
Proof. We have: $\frac{1}{2 \mathrm{~d}} \mathrm{~h}(z)=\frac{1}{2 \mathrm{~d}}\left(\left(\frac{1+\mathrm{t}}{1-\mathrm{t}}\right)^{\mathrm{d}}-1\right) \in \mathrm{S}^{*}$. Lemma 3 implies:

$$
\Re \exp \left(\int_{0}^{z} \frac{\left(\frac{1+t}{1-t}\right)^{d}-1}{t} d t\right)>0, z \in \mathrm{U} .
$$

On the other hand:

$$
\frac{z \mathrm{q}_{\mathrm{d}}^{\prime}(z)}{\mathrm{h}(z)}=\exp \left(\int_{0}^{z} \frac{\left(\frac{1+\mathrm{t}}{1-\mathrm{t}}\right)^{\mathrm{d}}-1}{\mathrm{t}} \mathrm{dt}\right) .
$$

These imply $\mathrm{q}_{\mathrm{d}} \in \mathrm{C}$, which means that $\mathrm{q}_{\mathrm{d}}$ is univalent. If the subordination $\mathrm{p} \prec \mathrm{q}_{\mathrm{d}}$ does not hold, then according to the Miller-Mocanu lemma it follows that there are two points, $z_{0} \in U$ and $\zeta_{0} \in \partial U$, and a real number $\mathrm{m} \in[1, \infty)$ such that

$$
\begin{aligned}
p\left(z_{0}\right) & =q_{d}\left(\zeta_{0}\right), \\
z_{0} p^{\prime}\left(z_{0}\right) & =m \zeta_{0} q_{d}^{\prime}\left(\zeta_{0}\right) .
\end{aligned}
$$

Since $h(U)$ is a starlike domain with respect to 0 , it follows that:

$$
\frac{z_{0} p^{\prime}\left(z_{0}\right)}{p\left(z_{0}\right)}=m \frac{\zeta_{0} q_{d}^{\prime}\left(\zeta_{0}\right)}{q_{d}\left(\zeta_{0}\right)}=\operatorname{mh}\left(\zeta_{0}\right) \notin h(U) .
$$

This contradicts the subordination $\frac{z \mathrm{p}^{\prime}(z)}{\mathrm{p}(z)} \prec h(z), z \in \mathrm{U}$. The obtained contradiction implies: $\mathrm{p} \prec \mathrm{q}_{\mathrm{d}}$.

Lemma 5 If $\mathrm{f} \in \mathcal{A}$ and

$$
\left|\arg \frac{z g^{\prime}(z)}{g(z)}\right|<\arctan \left(\frac{1}{2.273}\right), z \in \mathrm{U}
$$

then

$$
\left|\arg \frac{g(z)}{z}\right|<\frac{\pi}{6}, z \in \mathrm{U}
$$

Proof. The condition of the lemma is equivalent to

$$
\frac{z g^{\prime}(z)}{g(z)} \prec\left(\frac{1+z}{1-z}\right)^{\mathrm{d}}, z \in \mathrm{U} .
$$

Replacing in the previous lemma $p(z)=\frac{g(z)}{z}$, we get

$$
\frac{g(z)}{z} \prec q_{d}(z)=\exp \left(\int_{0}^{z} \frac{\left(\frac{1+t}{1-t}\right)^{d}-1}{t} d t\right), z \in u .
$$


Thus

$$
\left|\arg \frac{\mathrm{g}(z)}{z}\right| \leq \max _{\theta \in[-\pi, \pi]}\left|\mathfrak{I} \int_{0}^{1} \frac{\left(\frac{1+e^{i \theta} t}{1-e^{i \theta} t}\right)^{d}-1}{t} \mathrm{dt}\right|=v_{\mathrm{d}}\left(\theta_{\mathrm{d}}\right)<\frac{\pi}{6}, \quad z \in \mathrm{U} .
$$

In [1] the following theorem is proved.

Theorem 2 If $\mathrm{f} \in \mathcal{A}$, end

$$
\mathfrak{R} \frac{z g^{\prime}(z)}{g(z)} \geq\left|\mathfrak{I} \frac{z\left(z g^{\prime}(z)\right)^{\prime}}{g(z)}\right|, z \in \mathrm{U},
$$

then the following inequality holds:

$$
\mathfrak{R} \frac{z g^{\prime}(z)}{g(z)}>2.273\left|\mathfrak{I} \frac{z g^{\prime}(z)}{g(z)}\right|, \quad z \in \mathrm{U} .
$$

\section{The main result}

Theorem 3 If $\mathrm{g} \in \mathcal{A}$ satisfies (5), then

$$
\left|\arg \left(\mathrm{g}^{\prime}(z)\right)\right|<\frac{5 \pi}{17}, z \in \mathrm{U} .
$$

Proof. Inequality (6) is equivalent to

$$
\left|\arg \frac{z g^{\prime}(z)}{g(z)}\right|<\arctan \frac{1}{2.273}, \quad z \in \mathrm{U} .
$$

Thus according to Lemma 5 the inequality

$$
\left|\arg \frac{g(z)}{z}\right|<\frac{\pi}{6}, z \in \mathrm{U}
$$

follows. Summarizing we get

$$
\left|\arg g^{\prime}(z)\right| \leq\left|\arg \frac{z g^{\prime}(z)}{g(z)}\right|+\left|\arg \frac{g(z)}{z}\right|<\arctan \frac{1}{2.273}+\frac{\pi}{6}<0.92<\frac{5 \pi}{17} .
$$

If we could improve the previously proved result proving that $\left|\arg \left(g^{\prime}(z)\right)\right|<$ $\frac{\pi}{5}, z \in \mathrm{U}$, then it would follow that the next theorem is an improvement of Theorem 1. 
Theorem 4 If $\mathrm{f}, \mathrm{g} \in \mathcal{A}$ and

$$
\left|\arg \left(g^{\prime}(z)\right)\right|<\frac{\pi}{5}, z \in \mathrm{U},
$$

then the condition

$$
\mathfrak{R} \frac{f^{\prime}(z)}{g^{\prime}(z)}>0, z \in \mathrm{U}
$$

implies that $\mathrm{F}=\mathrm{A}(\mathrm{f}) \in \mathrm{S}^{*}$.

Proof. The conditions of the theorem imply

$$
\left|\arg f^{\prime}(z)\right| \leq\left|\arg \frac{f^{\prime}(z)}{g^{\prime}(z)}\right|+\left|\arg g^{\prime}(z)\right| \leq \frac{7 \pi}{10}, z \in \mathrm{U} .
$$

Using this result, we will prove that

$$
\left|\arg \frac{f(z)}{z}\right| \leq \alpha_{0}=\frac{50 \pi}{108}, z \in \mathrm{U} .
$$

To do this we rewrite inequality (11) in the following equivalent form:

$$
\frac{f(z)}{z} \prec\left(\frac{1+z}{1-z}\right)^{\frac{2}{\pi} \alpha_{0}}, z \in \mathrm{U} .
$$

If this subordination does not hold, then using Lemma 1 it follows that there are two points $z_{0} \in \mathrm{U}, \zeta_{0}=e^{i \theta_{0}} \in \partial \mathrm{U}$ and a real number $\mathrm{m}_{0} \in[1, \infty)$, such that:

$$
\begin{gathered}
\frac{f\left(z_{0}\right)}{z_{0}}=\left(\frac{1+\zeta_{0}}{1-\zeta_{0}}\right)^{\frac{2}{\pi} \alpha_{0}}=\left(i \cot \frac{\theta_{0}}{2}\right)^{\frac{2}{\pi} \alpha_{0}} \\
\left.z\left(\frac{f(z)}{z}\right)^{\prime}\right|_{z=z_{0}}=f^{\prime}\left(z_{0}\right)-\frac{f\left(z_{0}\right)}{z_{0}}=\frac{2}{\pi} m_{0} \alpha_{0} \zeta_{0}\left(\frac{1+\zeta_{0}}{1-\zeta_{0}}\right)^{\frac{2}{\pi} \alpha_{0}-1} \frac{2}{\left(1-\zeta_{0}\right)^{2}} \\
=\frac{2}{\pi} m_{0} \alpha_{0}\left(i \cot \frac{\theta_{0}}{2}\right)^{\frac{2}{\pi} \alpha_{0}-1} \frac{-1}{2 \sin ^{2} \frac{\theta_{0}}{2}} .
\end{gathered}
$$

Using these equalities, we deduce

$$
f^{\prime}\left(z_{0}\right)=\left(i \cot \frac{\theta_{0}}{2}\right)^{\frac{2}{\pi} \alpha_{0}}\left(1+i \frac{2}{\pi} \alpha_{0} \frac{m_{0}}{\sin \theta_{0}}\right) .
$$


Thus, if $\theta_{0} \in[0, \pi]$, then

$$
\left|\arg f^{\prime}\left(z_{0}\right)\right|=\alpha_{0}+\arctan \left(\frac{2}{\pi} \alpha_{0} \frac{m_{0}}{\sin \theta_{0}}\right) \geq \alpha_{0}+\arctan \left(\frac{2}{\pi} \alpha_{0}\right)>\frac{7 \pi}{10},
$$

and the case $\theta \in[-\pi, 0]$ is analogous to the previous one. If $\alpha_{0}=\frac{50 \pi}{108}$, then (12) holds, and this contradicts (10). The contradiction shows that inequality (11) holds.

We prove in the followings that

$$
\left|\arg \frac{\mathrm{F}(z)}{z}\right|<\alpha_{1}=\frac{3 \pi}{10} z \in \mathrm{U} .
$$

This inequality is equivalent to the subordination

$$
\mathrm{p}(z)=\frac{\mathrm{F}(z)}{z} \prec\left(\frac{1+z}{1-z}\right)^{\frac{2}{\pi} \alpha_{1}}=\mathrm{q}(z), z \in \mathrm{U} .
$$

If this subordination does not hold, then we use again Lemma 1 and we get that there are two points $z_{1} \in U, \zeta_{1}=e^{i \theta_{1}} \in \partial U$ and a real number $m_{1} \in[1, \infty)$, such that

$$
\begin{aligned}
\mathrm{p}\left(z_{1}\right) & =\mathrm{q}\left(\zeta_{1}\right), \\
z_{1} \mathrm{p}^{\prime}\left(z_{1}\right) & =\mathrm{m}_{1} \zeta_{1} \mathrm{q}^{\prime}\left(\zeta_{1}\right) .
\end{aligned}
$$

These equalities imply

$$
\begin{aligned}
\frac{f\left(z_{1}\right)}{z_{1}} & =z_{1} p^{\prime}\left(z_{1}\right)+p\left(z_{1}\right) \\
& =\left(i \cot \frac{\theta_{1}}{2}\right)^{\frac{2}{\pi} \alpha_{1}}-\frac{2}{\pi} \alpha_{1} m_{1} \times\left(i \cot \frac{\theta_{1}}{2}\right)^{\frac{2}{\pi} \alpha_{1}-1} \frac{1}{2 \sin ^{2} \frac{\theta_{1}}{2}} \\
& =\left(i \cot \frac{\theta_{1}}{2}\right)^{\frac{2}{\pi} \alpha_{1}}\left(1+i \frac{2}{\pi} \alpha_{1} \frac{m_{1}}{\sin \theta_{1}}\right) .
\end{aligned}
$$

If $\theta_{1} \in[0, \pi]$, then

$$
\arg \left(1+i \frac{2}{\pi} \alpha_{1} \frac{m_{1}}{\sin \theta_{1}}\right)=\arctan \left[\frac{2}{\pi} \frac{m_{1}}{\sin \theta_{1}}\right] \geq \arctan \left(\frac{2}{\pi} \alpha_{1}\right),
$$

and (14) implies

$$
\left|\arg \frac{f\left(z_{1}\right)}{z_{1}}\right| \geq \alpha_{1}+\arctan \left(\frac{2}{\pi} \alpha_{1}\right)>\frac{50 \pi}{108} .
$$


If $\theta \in[-\pi, 0]$, then the same inequality can be deduced. This contradicts (11) and the contradiction implies (13). Now we are able to prove that $F=A(f) \in$ $S^{*}$. Differentiating the equality $F=A(f)$ twice, we obtain

$$
F^{\prime}(z)+z F^{\prime \prime}(z)=f^{\prime}(z) .
$$

This can be rewritten using the notations $p(z)=\frac{z \mathrm{~F}^{\prime}(z)}{\mathrm{F}(z)}, \mathrm{P}(z)=\frac{\mathrm{F}(z)}{z g^{\prime}(z)}$ in the form

$$
\mathrm{P}(z)\left(z \mathrm{p}^{\prime}(z)+\mathrm{p}^{2}(z)\right)=\frac{\mathrm{f}^{\prime}(z)}{\mathrm{g}^{\prime}(z)} z \in \mathrm{U} .
$$

The conditions of the theorem imply that

$$
\mathfrak{R}\left[\mathrm{P}(z)\left(z \mathrm{p}^{\prime}(z)+\mathrm{p}^{2}(z)\right)\right]>0, z \in \mathrm{U} .
$$

We observe that (9) and (13) imply $|\arg (\mathrm{P}(z))|<\frac{\pi}{2}, z \in \mathrm{U}$ and this is equivalent to $\mathfrak{R P}(z)>0, z \in \mathrm{U}$. If $\mathfrak{R p}(z)>0, z \in \mathrm{U}$ is not true, then according to Lemma 2 it follows that there are two real numbers $x_{2}, y_{2} \in \mathbb{R}$ and a point $z_{2} \in \mathrm{U}$, such that $\mathrm{p}\left(z_{2}\right)=i x_{2}$ and $z_{2} \mathrm{p}^{\prime}\left(z_{2}\right)=y_{2} \leq-\frac{1}{2}\left(x_{2}^{2}+1\right)$. Thus the equality

$$
\mathrm{P}\left(z_{2}\right)\left(z_{2} \mathrm{p}^{\prime}\left(z_{2}\right)+\mathrm{p}^{2}\left(z_{2}\right)\right)=\mathrm{P}\left(z_{2}\right)\left(y_{2}-x_{2}^{2}\right)
$$

and $\mathfrak{R P}\left(z_{2}\right)>0$ imply that

$$
\mathfrak{R}\left[\mathrm{P}\left(z_{2}\right)\left(z_{2} p^{\prime}\left(z_{2}\right)+p^{2}\left(z_{2}\right)\right)\right] \leq 0 .
$$

This inequality contradicts (15), hence we deduce $\mathfrak{R} p(z)=\mathfrak{R} \frac{z \mathrm{~F}^{\prime}(z)}{\mathrm{F}(z)}>0, z \in \mathrm{U}$.

We end the paper stating a hypothesis.

Conjecture 1 We think that Theorem 3 and Theorem 4 can be improved in such a way that the obtained result would become an improvement of the second part of Theorem 1.

\section{References}

[1] A. Imre, P. A. Kupán, R. Szász, Improvement of a criterion for starlikeness, Rocky Mountain J. Math., 42 (2012).

[2] S. S. Miller, P. T. Mocanu, Differential subordinations theory and applications, Marcel Dekker, New York, Basel, 2000. 
[3] P. A. Kupán, R. Szász, About a condition for starlikeness, Ann. Univ. Sci. Budapest, Sect. Comp., 37 (2012), 261-274

[4] R. Szász, A counter-example concerning starlike functions, Stud. Univ. Babeş-Bolyai Math., LII. (2007), 171-172.

[5] R. Szász, An improvement of a criterion for starlikeness, Math. Pannon., 20/1 (2009), 69-77.

Received: 10 October 2013 